\title{
EXTRACTION OF URBAN FOOTPRINT OF BENGALURU CITY USING MICROWAVE REMOTE SENSING
}

\author{
Deepthi $\mathrm{R}^{1, *}$, Sudha Ravindranath ${ }^{2}, \mathrm{~K}$ Ganesha Raj $^{3}$ \\ ${ }^{1}$ Bharati Vidyapeeth Institute of Environment Education and Research, Pune, India - deepthi1911@gmail.com \\ ${ }^{2,3}$ Regional Remote Sensing Centre - South, National Remote Sensing Centre, \\ Indian Space Research Organisation, Bengaluru
}

Commission V, SS: Infrastructure and Development Planning

KEYWORDS: SAR (Synthetic Aperture Radar), Polarization, Urban, eCognition, SNAP

\begin{abstract}
Monitoring the urban development/change is of critical importance in planning the future infrastructure of a city. The use of satellite images in urban related studies has yielded in exemplary results. The city of Bengaluru, with high variation in urban landscape is most suited for this study. In this paper, the potential of the SAR imagery in understanding and characterizing the urban features is studied. The SAR images have unique characteristics such as double bounce and corner reflectors which are prominent in an urban landscape. The diverse urban features are characterised by comparing the graphs derived from the image statistics of temporal Sentinel -1 dual polarized data. For the generation of the urban footprint a rule based approach and an object oriented approach has been implemented in this study. The stack of coherence image and synthetic bands derived from image statistics of the VV polarization is used as the input image for the same. The final urban footprint is derived by the comparison of the output from both the methods. The results are authenticated with the urban footprint obtained by optical imagery of the same area for better understanding and improvement of the algorithm. The observations are made regarding the contribution of SAR in the study of urban features and the feasibility of implementation in the mainstream analysis.
\end{abstract}

\section{INTRODUCTION}

The urban footprint is defined as the overall extent of a city comprising of urban man made features including buildings and transportation networks. The urbanization is happening at a very fast pace in recent times and the growth of the city has to be monitored for better planning. The acquisition of the urban footprint from the satellite image is a well-established approach. With the advent of the SAR imaging techniques the utility of using the same has been explored and exemplary results have been obtained. Detection and analysis of settlement patterns, estimating population, and monitoring population migration are vital to accurately assess the impact of human activities on the environment. The SAR images can be used in the applications of settlement detection, population estimation, and assessment of the impact of human activities on the physical environment. The SAR image is very useful in distinguishing the buildings from the other features such as transportation networks (roads, railway) and barren land such as grounds in a city.

The property of corner reflectors and double bounce effects are very prominent in the SAR image of the urban landscape. The corner and the dihedral reflectors are a characteristic features which depict the buildings in the SAR image. All these factors are main contributors in understanding and analysis of urban features and buildings in SAR imagery.

\subsection{STUDY AREA}

Bengaluru city is located in the state of Karnataka in the southern part of India.

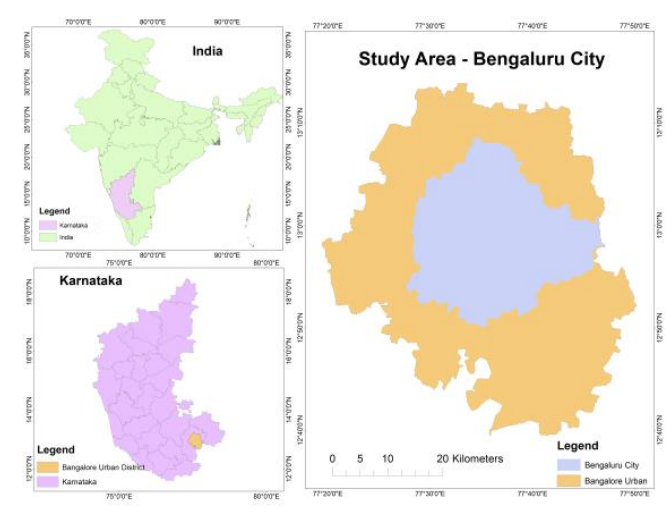

Figure 1: Study area map of Bengaluru city

\footnotetext{
* Corresponding author
} 
It is situated at an elevation of $914.4 \mathrm{~m}$. The city spans about $712 \mathrm{Km}^{2}$ in area. The population census of the city in 2018 was found to be around 10 million .Bengaluru

city is famously known as the silicon valley of India and has employsaround $35 \%$ of India's 2.5 million IT professionals. It is also fondly known as the garden city due to the greenery and well known parks such as Lalbagh and Cubbon Park.

\subsection{RESEARCH QUESTIONS}

The aim of this study is to understand the potential of microwave images to study the urban features. The properties of the urban features in the $\mathrm{VV}$ and $\mathrm{VH}$ polarization are studied by statistical analysis. The SAR image is very useful in distinguishing the buildings from the other features such as transportation networks (roads, railway) and barren land such as grounds in a city. The property of corner reflectors and double bounce effects are very prominent in the SAR image of the urban landscape.The following research questions are considered by the above stated understanding:

1. What is the statistical analysis and characterization of responses of urban features in SAR?

2. What is the accuracy of an urban footprint generated from SAR data and the optical data of Bengaluru city and comparison of the same?

\subsection{LITERATURE REVIEW}

Anthony Lewis (1968) et.al [1] this paper briefly discusses about the various polarization of RADAR imaging and the influence of the same on land use features. The paper gives a clear cut idea on which polarization should be used for the studying different landscapes like urban, rural, transportation networks etc. In rural environments cross-polarized imagery $(\mathrm{HV}, \mathrm{VH})$ is generally better for defining cultural objects such as farmsteads and transportation networks. Whereas in urban the buildings are more clearly delineated in the like polarization than otherwise. The orientation of the building, roof material and height of the building are contributing factors in the image. For the detection of the roads and railway both cross and like polarization have given good results however this varies with the morphology of every study area.

Fabio Dell'Aqua and Paolo Gamba( 2003) et.al [2] In this paper different textural parameters were analyzed for a single date SAR image. The texture parameters such as contrast, correlation, dissimilarity, entropy, mean, homogeneity and second moment. The various texture parameters values were analyzed for different urban features. The urban density maps were derived after characterizing the urban features by texture. Multitemporal SAR data was analyzed for the same and the density maps were derived. The consistency was observed in the density maps. This paper gives a clear understanding about the influence of the texture parameters in the SAR image and implementing it in the classification

algorithms.
Floyd M Henderson (1997) et.al[3]this paper focuses in understanding the factors influencing the SAR imagery of the urban features. The important categories that influence the settlement visibility in the SAR image are category or the type of building function, construction material, surrounding land cover, wavelength polarization look direction and incident angle. This paper explains the practical utility of using SAR images in the extraction of urban features i.e. the urban and socioeconomic indicators can be derived from the SAR images which are used by planning commission.

ThomasEsch (2010) et.al [4] this paper presents an approach toward semi-automated detection of built-up areas based on single polarized TSX images. The method includes specific pre-processing of the SAR data and an automated image analysis procedure. The pre-processing focuses on analysis of local speckle characteristics in order to provide a texture to highlight built-up areas. Object - Oriented image analysis is used to automatically extract the built-up areas using the texture and the intensity layers.

D G Corr (2003) et.al [5] this paper discusses the approach of object oriented image analysis and segmentation techniques on SAR images. The methodology utilized is the through the generation of coherence images from temporal complex SAR images. This was complemented by the information from polarimetric decomposition of the data into entropy and anisotropy. To perform further classification of features as urban fuzzy membership classes were defined within the eCognition software. The prominence of the relationship between the building orientation and radar look direction is highlighted with substantial ground data. Considering the above stated factors a hierarchical approach has been implemented in this paper for the extraction of urban area.

Junichi Susaki (2015) et.al [6] this paper compares the potential of extracting the urban areas from $L$ band and $X$ band fully polarimetric SAR images. For the analysis of $\mathrm{L}$ band images the POA (Polarization Orientation Angle) volume scattering $(\mathrm{Pv})$ and total power $\left(\mathrm{T}_{\mathrm{p}}\right)$ data is used for classification. For the analysis of $\mathrm{X}$ band images a thresholding approach of the total power and volume scattering values is used. It was found that in mountainous region the $\mathrm{X}$ band had poorer returns than the $\mathrm{L}$ band. It was concluded that theusage of $\mathrm{T}_{\mathrm{p}}$ and $\mathrm{Pv}$ for the classification of urban features from SAR data.

Andy Reiu (2017) et.al [7]the aim of this study was to see how precisely the urban areas can be mapped using interferometric coherence on Sentinel -1 satellite imagery with different conditions. The conditions are the time separation of 3 months and 1 year, ascending and descending orbits, winter and summer data. This study has used the image sets off Sentinel -1 SLC data. Another important aspect of this study is the classification of urban and non-urban areas that would lead to time-saving improvements when using manually derived rules for building detection. The major conclusion was the accuracy of the urban maps extracted from the coherence image; it showed that a time separation of 3months has higher accuracy than 1 year. 


\section{MATERIALS AND METHODS}

\subsection{DATA COLLECTION}

\subsubsection{Satellite Images}

Sentinel - 1SLC SAR data and Sentinel - 2 optical data both are acquired with a spatial resolution of $10 \mathrm{~m}$ was used in the course of this study which includes the following three datasets:

a) Sentinel - 1A SLC SAR Image dated $13^{\text {th }}$ October 2017.

b) Sentinel -1A SLC SAR Image dated $5^{\text {th }}$ January 2018.

c) Sentinel - 2AOptical Image dated 12th January 2018.

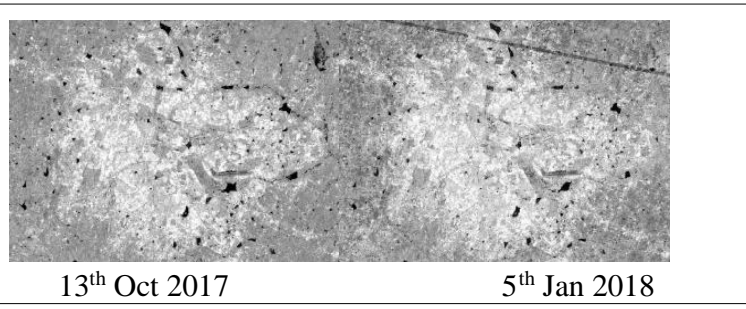

Figure 2: Sigma Naught Sentinel -1A SAR Images

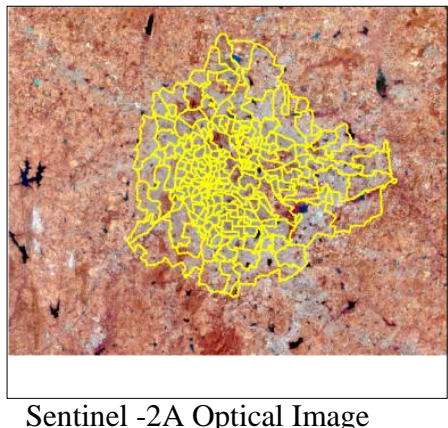

Figure 3: Satellite Image and GIS generated ward boundary referred in the Study

\subsubsection{Ancillary Data}

1. Ward Boundary in the form of attribute table and vector layer generated using GIS tools

2. Vector layers of transportation networks (roads and railway lines) of the city.

\subsection{METHODOLOGY}

\subsubsection{Characterization of responses of urban features in SAR Data}

The statistical approach is taken to understand the backscatter response from the urban features. The
Backscatter is dependent on the dielectric constant and the surface roughness of the feature at interest. The mean, max, min and standard deviation are considered in the statistical analysis. The features considered in this methodology are barren, parks, lakes, lakes with weed, agricultural patch, transportation networks (roads and railway) and types of built-up. For each of the feature the statistics are analysed and conclusions are drawn on as to why the values of the feature are so. A graph is derived from the statistical analysis for each of the polarization (VV/VH for Sentinel - 1). The graph is a clear representation of the interaction between the features of interest and the polarization. The values derived from the above analysis are used in the next objective to extract the urban footprint and also in the final objective in the feasibility in delineating individual buildings.

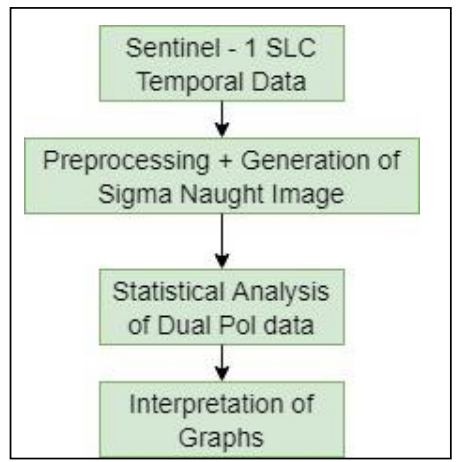

Figure 4: Methodology Flowchart of first objective

\subsubsection{Generation of urban footprint using inputs from SAR and Optical data.}

For the extraction of the urban footprint from SAR data dual date Sentinel - 1 SLC (Single Look Complex) is used. The SLC data is the complex data which contains the phase and amplitude intensity. Single Look Complex (SLC) products are images in the slant range by azimuth imaging plane, in the image plane of satellite data acquisition. Each image pixel is represented by a complex (I and Q) magnitude value and therefore contains both amplitude and phase information. The phase information in the SLC images is used to generate the coherence image. The prerequisite of the creating a coherence image is a set of Sentinel - 1 SLC products with any measurable time difference. Generating the coherence image is process intensive the methodology for the same is written the annexure. The synthetic bands are generated using the understanding and the conclusion derived from the first objective of the statistical analysis. The synthetic bands generated are of the mean and the difference statistics. The three images of coherence and the two synthetic bands are layer stacked to make a combined image. To generate the urban footprint a rule based approach and an object oriented approach is considered. The rule based approach uses a conditional algorithm considering the values of coherence and mean. It is found that in urban areas with features such as buildings the coherence values are generally high or nearing to 1 , since the features rarely change with time. 
The mean values of Sigma naught considered for urban features are shown to be mostly ranging in positive values. The footprint using the rule based approach is derived from the SNAP software. For the object oriented approach a segmentation algorithm is run with a nominal shape and compactness value on the layer stacked image. A thresholding algorithm is run with conditions on coherence and mean image. The footprint is derived as a vector. The final urban footprint is derived by taking inputs from both the procedure.

The urban footprint is generated using the Sentinel - 2 optical data for comparison and improvement of the results derived from SAR data. The Sentinel -2 optical data has the SWIR band which is very useful in the monitoring of urban features. An urban index is used using the SWIR and NIR band to delineate the urban features. The urban footprint derived from all the three methods are compared and the justified.

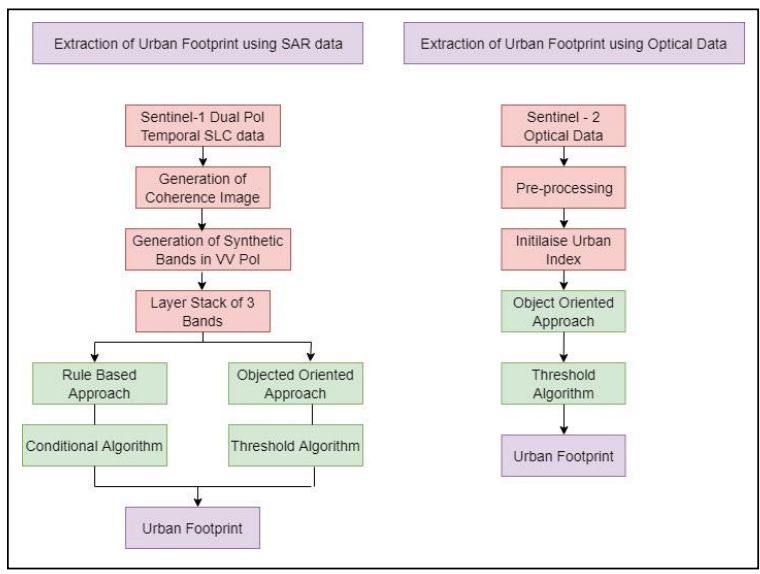

Figure 5: Methodology Flowchart of second objective

\section{RESULTS AND DISCUSSIONS}

\subsection{Characterization of responses of urban features in SAR Data}

The below are the graphs derived from the analysis done from the first objective. The graph derived from the statistical analysis that is implemented in this study for the characterization of the urban features gives a clear idea about the interaction of SAR wavelength and polarization of land features.

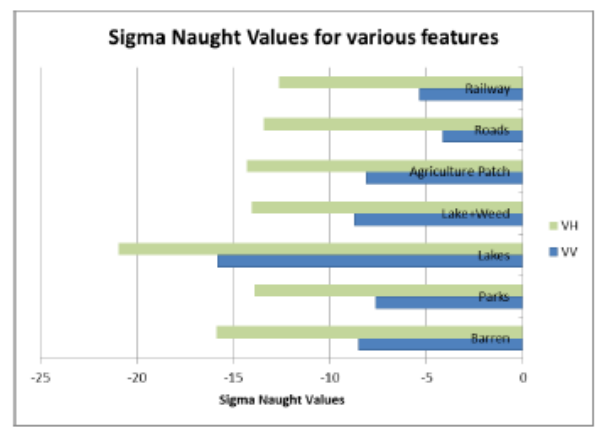

Figure 6: Sigma Naught values of various features
The graph derived from the statistical analysis of urban features except buildings using the 10m Sentinel -1 dual pol data. The features considered here are barren, parks, lakes, lakes with weed, agricultural patch, transportation networks (road and railway). The sigma naught values are all negative for these features as they do not reflect the incident energy more. The above features mostly act as smooth surfaces to the incoming incident energy and hence the specular reflection occurs. Since the backscatter values for all the above mentioned features are negative there is a chance in overlap of classification of features. Hence other properties such as surface roughness should be considered to distinguish between the features.

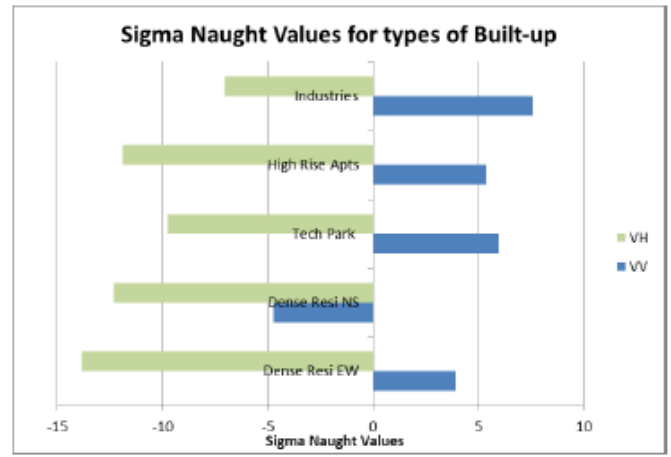

Figure 7: Sigma Naught Values of Built-up

The primary observation in this graph is the positive values for the VV polarization data of different built-up areas (The backscatter values are most likely to be high in like polarized return than cross polarized return; this is due to the fact that when the transmit polarization is vertical/horizontal then generally the land features return the incident energy in the same polarization as received, there has to be a depolarizer as the target to give a bright return in the cross polarization which is a rare phenomenon in land use features, hence the negative values of cross polarized data). This is the graph derived from the statistical analysis of different types of built-up. The type of built up considered here are industries, high rise apartments, tech parks, dense residential complexes with have a North-South orientation and dense residential complexes having an east west orientation. In the analysis of interaction of SAR incident energy with the urban features the major characteristics are the double bounce effects and corner reflections. The RCS of the double bounce is dependent on the area of the building wall and its height. The higher the building, the stronger the double bounce. Hence the sigma naught values for Tech Park, high rise apartments and industries have very high positive values ranging from $5.32-7.68 \mathrm{db}$. Since the industries represent a sparser distribution and with metallic roofs the double bounce scattering are generally less for this feature. However, since the data considered here is of $10 \mathrm{~m}$ resolution the industries appear to be in a cluster giving a combined high backscatter. The orientation of the building with respect to the direction of 
illumination is very important in understanding the backscatter sigma naught values. In the above graph it is clear that the dense residential area with EW orientation have higher sigma naught values than the NS oriented buildings. The EW oriented buildings are in the direction (parallel) of the radar illumination and the NS oriented buildings are perpendicular to the direction of illumination. The RCS of the buildings parallel to the direction of illumination is small and thus act as sharp corner reflectors resulting in a positive backscatter value. The buildings with perpendicular orientation to the direction of illumination have more building area exposed towards the incoming radiation. Since the buildings are all aligned close to each other this will result in the NS oriented building to behave as a specular reflector thus dissipating all the energy.

\subsection{Generation of Urban Footprint with inputs from SAR and optical data}

The below is the description of images and the urban footprint derived from the analysis of the second objective. The first approach used for deriving the urban footprint using the Sentinel - 1 SAR data is obtained by the generating coherence maps using two date Sentinel -1 single look complex data and synthetic bands using statistical analysis. The phase information contained in the complex data is used to generate the coherence image. The statistical information of mean and difference is used to generate the synthetic bands of mean and difference image. A layer stack of the three bands are used and a rule based approach is implemented using conditional algorithm to extract the urban footprint. The same is performed using OBIA.

The accuracy assessment is a very important part of the image classification process. It is a statistical measure of the exactness of the classified features. Accuracy assessment is performed using various methods based on the results obtained and the objective of the study. The method of calculating the accuracy assessment in this study is done using a grid method. The output is divided into grids and random numbers are generated as per the number of grids. The grids are chosen as per the random number. The ground truth points (GT's) are taken within the grid created on the output derived from the first two objectives. The result from the first objective is the urban footprint of the Bengaluru city derived from two different methodologies using SAR image. The derived footprint is first checked with the same generated using the optical Sentinel -2 data. The accuracy is checked for the three urban footprints using the above mentioned grid method. The accuracy of the footprint derived from the object based approach is approximately $68.31 \%$ while the rule based approach gave an accuracy approximated to $71 \%$. This lead to the conclusion that the OBIA procedure has a satisfactory outcome on medium resolution SAR data. The rule based approach gave a higher accuracy as it was a pixel based approach and the land use features are less confused in a SAR image in this approach. The accuracy of the urban footprint derived from the optical data is approximately $72.37 \%$. The optical data proved to have higher accuracy due the availability of the SWIR band in the Sentinel -2 data. The analysis of urban in SWIR band has always given exemplary results.
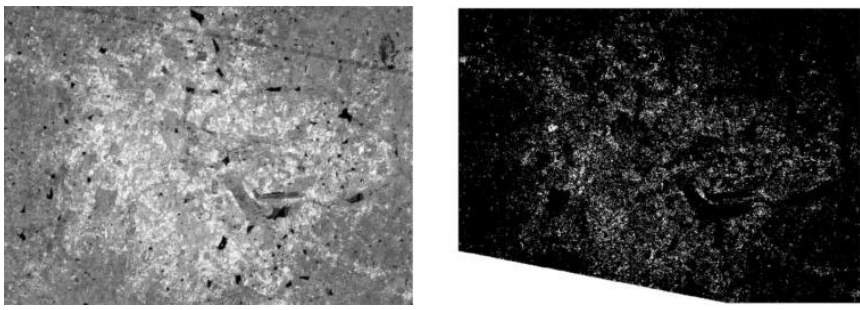

Figure 8:Mean Image

Figure 9: Urban Footprint
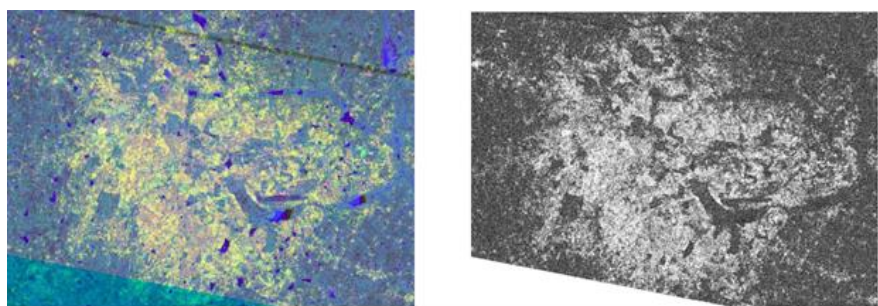

Figure 10: Layer Stack Image

Figure 11: Coherence Image

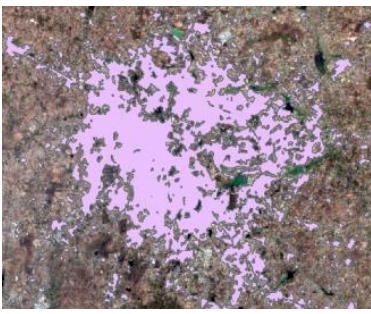

Figure12:Urban Footprint from Optical dataset

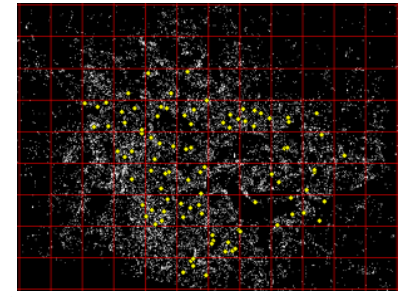

Figure 13:Accuracy Assessment using grid method

\section{CONCLUSIONS}

This project gives a detailed understanding that the like polarization $(\mathrm{VV} / \mathrm{HH})$ gives the optimum results in analysing urban features. In this study since the data was of coarse resolution the rule based approach gave better results as compared to the object based approach. The graphs explain the influence of dielectric constant and surface roughness on the backscatter co-efficient sigma naught. The values from the statistical analysis are used to extract the urban footprint with inputs from the coherence image. The urban footprint is generated using the optical data for comparison and improvement of the algorithm. The urban footprint derived from the SAR coherence image is a very robust method to understand the urban sprawl. It is a very fast process that clearly delineates the built - up area of the city. The accuracy of the pixel based approach is approximately $71 \%$ and the OBIA was $68.31 \%$. This method is not algorithm or process intensive as it is in the processes using optical data. The area of the urban footprint extracted from SAR dataset is $762 \mathrm{~km}^{2}$ and that from optical is $810 \mathrm{~km}^{2}$. The area obtained in optical is more due to discrepancy caused in distinguishing barren and built-up. This method can further be used in urban studies in creating urban growth models. 


\section{FUTURE SCOPE OF STUDY}

The SAR images have great potential in urban studies and can give exemplary and quick results. This study can be extended for full polarization and hybrid polarization SAR datasets. By utilising the VV, VH, HH and $\mathrm{HV}$ bands novel urban extraction indices can be generated that would give results with higher accuracy. Also, the dual aspect images can be considered as it would enhance the understanding of impact of radar illumination direction on building orientation. Interferometric analysis of the complex SAR images will also provide highly valuable information through which the $3 \mathrm{D}$ rendering of the buildings can be made possible.

\section{ACKNOWLEGDEMENT}

The authors thank Director, NRSC and CGM RRSCs for encouragement. We thank Bangalore Municipal Corporation for providing necessary data for carrying out our research. Authors are thankful to Dr. V Poompavai, Scientist/Engineer, RRSC-S Bengaluru andBharatiVidyapeeth Institute of Environment Research and Educaation for providing the necessary guidance. Weextend our sincere thanks to the technical as well as non-technical staff of RRSC-S for providing moral support during the course of our work.

\section{REFERENCES}

1. Anthony J Lewis et al(1968) "EVALUATION OF MULTIPLE POLARIZED RADAR IMAGERY FOR THE DETECTION OF SELECTED CULTURAL FEATURES", interagency report, Geological survey for NASA[1]

2. Fabio Dell'Acquaet al(2003) "TEXTURE BASED CHARACTERIZATION OF URBAN ENVIRONMENTS ON SATELLITE SAR IMAGES",IEEE Transactions on Geoscience and remote sensing ,Volume 41, Number 1,January 2003[2]

3. Flyod M Hendersonet al(1997)"SAR APPLICATION

IN HUMAN SETTLEMENT

DETECTION,POPULATION ESTIMATION AND URBAN LAND USE PATTERN ANALYSIS: A STATUS REPORT" IEEE Transactions on Geoscience and remote sensing, Volume 34, Number 1,January 1997[3]

4.T.Esch et al(2010)"DELENIATION OF URBAN FOOTPRINTS FROM TERRASAR-X DATA BY ANALYZING SPECKLE CHARACTERISTICS AND INTENSITY INFORMATION" IEEE Transactions on Geoscience and remote sensing, Volume 48, Number 2,Febraury 2010[4]
5.DCorret al(2003) " CLASSIFICATION OF URBAN SAR IMAGERY USING OBJECT ORIENTED TECHNIQUES"IEEE Transactions on Geoscience and remote sensing, Volume 17, Number 3,Febraury 2003[5]

6.Junichi Susakiet al(2015) "COMPARISION OF URBAN AREAS EXTRACTED BY USING L-BAND AND X-BAND FULLY POLARIMETRIC SAR IMAGES" IEEE Transactions on Geoscience and remote sensing, Volume 31, Number 5,January 2003[6]

7. Andy Reiuet al (2017) “ CLASSIFICATION OF URBAN AREAS FROM SENTINEL - 1 COHERENCE MAPS", Master Thesis, Institute of Physics, University of Tartu [7] 